\title{
ELECTRON CYCLOTRON HEATING ON THE ISX-B TOKAMAK*
}

O. C. Eldridge, A. C. England, R. M. Gilgenbach,

K. F. Hackett,, A. G. Kulchar, $a$ C. M. Loring, and J. B. Wilgen

Oak Ridge National Laboratory

Oak Ridge, Tennessee, U.S.A.

\begin{abstract}
One experiment on electron cyclotron heating on the Impurity Study Experiment (ISX-B) tokamak has been completed and a second experiment is in progress. In the first experiment, with a gyrotron producing $100 \mathrm{~kW}$ at $35 \mathrm{GHz}$, a heating efficiency greater than $60 \%$ was achieved. Unpolarized radiation was launched from the high field side of the tokamak, heating electrons at cyclotron resonance in the center of the plasma. From laser scattering and thermal emission at the second gyroharmonic, the electron temperature, initially $850 \mathrm{eV}$, increased to $1250 \mathrm{eV}$ at the end of a 15-ms pulse. The second experiment is now in progress, with $180 \mathrm{~kW}$ at $28 \mathrm{GHz}$ and a capability of 100 -ms pulses. A comparison between polarized and unpolarized radiation, modification of the plasma current profile, and a critical comparison with theory are planned. In this paper, experiments on preionization at the upper hybrid resonance are reported. A plasina with an electron temperature of $10 \mathrm{eV}$ was produced, which is not high enough to reduce impurity radiation. The resistive part of the loop voltage was reduced by preionization. Plans for an experiment to measure electric current induced by electron cyclotron heating are presented, along with a review of the theoretical basis of the current drive.
\end{abstract}

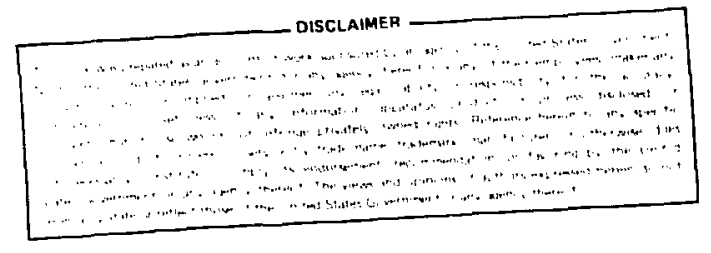

\footnotetext{
University of Tennessee, Knoxville, Tennessee, U.S.A. $\dot{D}_{\text {JAYCOR, InC., Alexandria, Virginia, U.S.A. }}$ Massachusetts Institute of Technology, Cambridge, Massachusetts, U.S.A. * Research sponsored by the Office of Fusion Energy, U.S. Department of Energy, under contract $w-7405-e n g-26$ with the Union Carbide Corporation.
} 


\section{INTRODUCTION}

Experiments with electron cyclotron heating (ECH) on the I5X-8 tokamak were planned after successful Russian experiments on FT-l l and TM- $3^{2}$ and after the development of a long pulse gyrotron by Varian Associates. The long pulse experiment has been delayed by technical problems, but experiments with ECH have been performed with a $35-\mathrm{GHz}$ gyrotron developed at the Naval Research Laboratory (NRL). ${ }^{3}$ Experimental results for bulk heating and for preionization are reported here. Theoretical predictions for planned experiments to induce toroidal electric currents in association with $\mathrm{ECH}$ and for profile modification are also summarized.

\section{ELECTRON CYCLOTRON HEATING AT $35 \mathrm{GHz}$}

Experiments ${ }^{4}$ performed on ISX-B with a 35-GHz gyrotron resulted in an ohmic heating power (100-150 kW) and energy confinement time ( $9 \mathrm{~ms}$ ) at $\bar{n}_{e}=1.0 \times 10^{13} \mathrm{~cm}^{-3}$ comparable to the $r f$ power $(100-150 \mathrm{~kW})$ and the gyrotron pulse length ( $\$ 16 \mathrm{~ms})$. The frequency of the gyrotron was such that the ECH resonant surface could be located at any desired minor radius. This enabled plasma heating at the plasma center or off axis. Electron densities in these experiments were kept at $51.5 \times 10^{13} \mathrm{~cm}^{-3}$, which corresponds to cutoff for the 0 mode radiation. Microwave power was transmitted to the tokamak in the $T E_{01}$ circular electric mode in oversized (6-cm-ID) copper waveguide. Unpolarized microwave power was injected from the midplane of the high field side of ISX-B to avoid the cutoff of the $X$ mode. The angle of incidence was $45^{\circ}$ with respect to the magnetic field at the plasma edge. As shown schematicaliy in Fig. 1 , the launching antenna was located in the shadow of the inner 1 initer inside the tokamak and consisted of an open circular waveguide and a reflecting plate.

\section{Predictions}

Calculations 5 indicate that for a device like ISX-B, almost complete single pass absorption of the $X$ mode radiation will occur if the radiation is iaunched from the high field side at an angle of $s 60^{\circ}$ from the toroidal field direction. This is shown in Fig. 2. At high densities, the range of acceptable angles decreases due to refractive effects. For the 0 mode, incomplete ( $40 \%$ ) absorption is calculated for a single pass with angles near normal to the field direction preferred.

\section{Temperature and Dens ity Measurements}

Electron temperatures were measured by laser Thomson scattering and two superheterodyne receivers that measured the second hamonic cyclotron emission at $\sim 70 \mathrm{GHz}$ and $\sim 58 \mathrm{GHz}$. The experimental results from the Thomson scattering are shown in Fig. 3. Note that the central electron temperature rises with the 80-kH, 10-ms pulse by 2400 eV and the temperature profile becomes more peaked. The density shows a decrease that is at present not understood. It is too large to be accounted for by the diminution of the Ware pinch due to the lower loop voltage. 
Figure $4(a)$ shows the time behavior of the temperature (measurement by a second harmonic radiation detector), loop voltage, and density with a longer (16-ms) pulse at $80 \mathrm{~kW}$. The line-averaged density decreases 215\%, the loop voltage decreases $260 \%$, and the central electron temperature rises $4.50 \%$.

Figure $4(b)$ shows the result of comparing the second harmonic temperature measurement at $70 \mathrm{GHz}$, the central laser temperature, and a calculation using all empirical transport code. Good agreement is found betireen all three. A calculation of the power balance gives a measured efficiency of $260 \%$ while the theoretical efficiency for single pass absoptotion is $270 \%$.

The decay time of the second harmonic detector signal after cessation of the microwave power typically has an w3-ms e-folding time with indications of a longer tail. The computer simulation also has a 3-ms decay time, which is much shorter than the energy containment time. The shortened decay time is caused by two effects. The localized heating produces largar temperature gradients and an initial fast decay. Also. the ohmic heating increases as the temperature drops so that the equilibrium tengarature is reached in a shorcar time.

In Fig. 5 , the central electron temperature is shown as a function of the microwave power level. Note the linear relation as indicated both by the laser medsuremert and the second harmonic detection. The measurement shows a slope $01 \% 5.5 \mathrm{eV} / \mathrm{KW}$ at $\mathrm{n}_{\mathrm{e}}=10^{13} \mathrm{~cm}^{-3}$ for a plasma volume of $1.3 \mathrm{~m}^{5}$.

The ion temperature, as measured by a charge exchange analyzer, did not change. Ion heating is not expected because the electron-ion equilibration time is much longer than the energy confinement time and the microwave pulse length.

\section{Anomalies}

The abrupt decrease in electron density and the equally fast recovery after the microwave pulse are not understood. Tentatively one may say that they are associated with changes in the plasma equilibrium, and that they present some slight evidence of an induced plasma current. The signal from the soft $x$-ray detector is also anomalous, with a delayed response. Since this signei depends on electron density and temperature, one may interpret these data as evidence of changes in the plasma equilibrium.

\section{PREIONIZATION}

\section{Experiments}

Previous calculations ${ }^{6}$ indicated that substantial savings in transformer webers and initial breakdown vol tage could be achieved wi th pretonization. However, recent results ${ }^{7}$ on $F T-i$ do not substantiate this theoretical result. Preionization experiments ${ }^{8}$ were performed on is $X-B$ with the same configuration mentioned earlier. Two types of experiments were performed. First, microwave power was injected into isX-B with only the toroidal magnetic field applied and the usual gas fllling. The preionized plasma density, temperature, and confinement time were investigated. While the toroidal magnetic field was varied 
froin shct to shot, time-resolved density profiles were obtained from a vertical microwave interferometer. It appears that the density initially peaks at $12.5 \mathrm{kG}$, the resonant field, but quickiy spreads to fill the vacuum chamber. After $2-3 \mathrm{~ms}$, a peak appears at a lower field; this may be due to absorption at the upper hybrid resonance. Comparisons between vertical and horizontal interferometers suggest a plasma with a vertical elongation of $3: 1$.

High speed ( $4300-4 s / f r a m e)$ motion pictures also showed a breakdown at the cyclotron resonance and a rapid motion outward to a bright region that appears to be the upper hybrid resonance position. Spectrosecpic measurements of impurities indicated a bulk temperature of $11-13 \mathrm{eV}$, which is confirmed approximately by a Langmuir probe $(8-10 \mathrm{eV})$ after the rf pulse ends and by a gridded analyzer (8-10 eV). The gridded analyzer also showed a higher temperature ( $250 \mathrm{eV}$ ) near the calculated upper hybrid region. The preionized plasma was quite stable with an e-folding time after power turn-off of a $10 \mathrm{~ms}$. This indicates a low electron temperature $(210 \mathrm{eV})$ if the decay is due to gradient $B$ drift.

In the second type of preionization experiment, applying the microwave power a fely militisecends before the loop voltage was applied showed the effect of flux economy. Without ECH preionization, there was a large short-lived voltage spike at the time the capacitor bank was fired. With ECH preionization, this voltage spike was $E l$ iminated and the volt tage level immediately after was also reduced. The plateau voltage reduction after the inttial spike was $40 \%$, and about $40 \%$ of the normal flux expended in the first $2 \mathrm{~ms}$ was saved. The initial rate of current rise was increased and Thomson scattering measurements showed a higher electron temperature during the current rise phase of the discharge. Almost all of the initial loop voltage with preionization was due to the inductive term, $L(d I / d t)$. Without preionization, there was a large resistive component. The initial emission from 0 II, 0 III, 0 IV, and Fe XIV lines was reduced. However, their amplitudes later in the discharge were unchanged.

\section{Interpretation}

The electron temperature achieved was quite low, so there is some enhanced loss of energy from the electrons. One may assume that this loss is the radiative excitation of impurity atoms. In this case, increased power into the localized heating zone near the upper hybrid resonance should produce a large volume of hot plasmo. There is a possibility that the short energy containment time is caused by the interchange instability at the outboard side of the plasma, near the wall.

\section{CURRENT ORIVE WITH ECH}

According to a recent calculation, 9 toroidal current is induced whenever there is strong absorption of microwaves in a tokamak. Although ECH changes the perpendicular energy of the electrons, an anisotropy in the parailel velocity is produced by the Doppler shift. An electron drifting toward the source interacts at a iarger magnetic field. With 
very strong absorption, radiation incident from the high field side damps away before reaching the cold electron resonant surface, so a unidirectional population of electrons is heated. This anisotropic electron distribution gains momentum by collisions with ions, since hot electrons have a smaller collision frequency. Radiation incident from the high field side should induce a current along the magnetic field in the direction of the ray.

The total current induced is predicted to be

$I_{E C H}(A)=\frac{3.83(10)^{13 P_{0}}(W) T(k e V) Q_{J}}{R(m) n\left(\mathrm{~cm}^{-3}\right) \ln \Lambda}=\frac{e}{m} \frac{P_{C}}{R \bar{v}_{e i}} \sqrt{\frac{\pi}{T}} Q_{J}$,

where $P_{0}$ is the incident power. The function $Q_{j}$ is plotted in Fig. 6 , in terms of the optical depth $n$. One sees that current drive requires a stronger interaction than is needed for complete absorption. In Fig. 7 , the spatial dependence of the induced current and power density is

plotted, with $\Delta=R \cos \psi \sqrt{T / m c^{2}}$. For this case the velocity of the electrons being heated is peaked at 1.5 times the thermal velocity. For $n=10$, a minute amount of wave energy is transmitted.

Figure 8 shows the case of weak absorption, with $n=1$, corresponding to $63 \%$ absorption. One sees that there is a negative current on the far side of resonance. The opposing currents cancel when averaged over drift surfaces.

For the experiment in.progress on ISX-B, assuming $100 \mathrm{~kW}$ of power in the extraordinary mode at an angle of $30^{\circ}$ to the magnetic field and an electron temperature of $1.5 \mathrm{keV}$, one expects $30 \mathrm{kA}$ of induced current. since the machine operates in a constant current mode, the loop voltage will be monitored for differences between coinjection and counterinjection. For the experiment at $35 \mathrm{GHz}$, about $10 \mathrm{kA}$ of current should have been induced, but any change in loop voltage was masked by increased conductivity.

This current source has been investigated with the equilibrium code devised by $J$. T. Hogan, which includes field lifie reconnections according to the Kadomtsev model. One sees sawtooth oscillations and fairly drastic changes in the plasma equilibrium. 


\section{REFERENCES}

1. The latest report of this experiment is given by D. G. Buliginskii et al., in Eroc. 9th European Conf. on Controlled Fusion and Plasma Physics (Oxford, September 1979), p. 547.

2. V. V. Alikaev et al., Fiz. Plazmy 2. 390 (1976) [Sov. J. Plasma Phys. 2, $212(1976)]$.

3. M. E. Read et al., in International Electron Devices Meeting (IEEE Publications, Piscataway, New Jersey, 1979), p, 172.

4. R. M. Gilgenbach, Phys. Rev. Lett. 44, 647 (1980).

5. 0. C. Eldridge et al., Electron Cyclotzon Heating in Tokemaks, ORNL/TM-6052, Oak Ridge National Laboratory, Oak Ridge, Tennessee (1977); submitted to Nucl. Fusion.

6. Y-K. M. Peng et al., Nucl. Fusion 18, 1489 (1978).

7. D. G. Buliginskii et al., Joffe Physical-Technical Institute Report 671 , 1979; submitied to Fiz. Plazmy.

8. R. M. Giigenbach et al., "Electron Cyclotron/Upper Hybrid Resonant Preionization in the ISX-B Tokamak" submitted to Nucl. Fusion.

9. 0. C. Eldridge, On Currents Induced in Plasmas by Cyclotron Heating, ORNL/TM-7503, Oak Ridge National Laboratory, Oak Ridge, Tennessee (in press); to be subnitted to Phys. Rev. Lett. 
FIGURE CAPTIONS

Fig. 1. Schematic diagram showing the microwave antenna in a crosssectional view of ISX-B.

Fig. 2. Absorption in the ISX-8 tokamak plotted as a function of density for several angles of incidence.

Fig. 3. (a) Electron temperature and (b) density imediateiy before and at the end of the ECH pulse as measured by lasar Thomson scattering.

Fig. 4. (a) Time behavior of the electron density, loop voltage, and 70-GHz second harmonic amplitude for the ECH experiment with a 16-ms pulse. (b) An expanded view of the second harmoric signal (solid line). Also shown are central temperatures measured by Thomson scattering and (dotted) a model calculation for the central temperature using a one-dimensional transport code.

Fig. 5. Central electron temperature vs microwave power for ISX-B.

Fig. 6. The normalized current $Q_{j}$ and single pass absorption as a function of optical depth.

Fig. 7. Heating and induced current profiles as a function of distance from resonance for strong absorption.

Fig. 8. Heating and current profiles for weak absorption. 

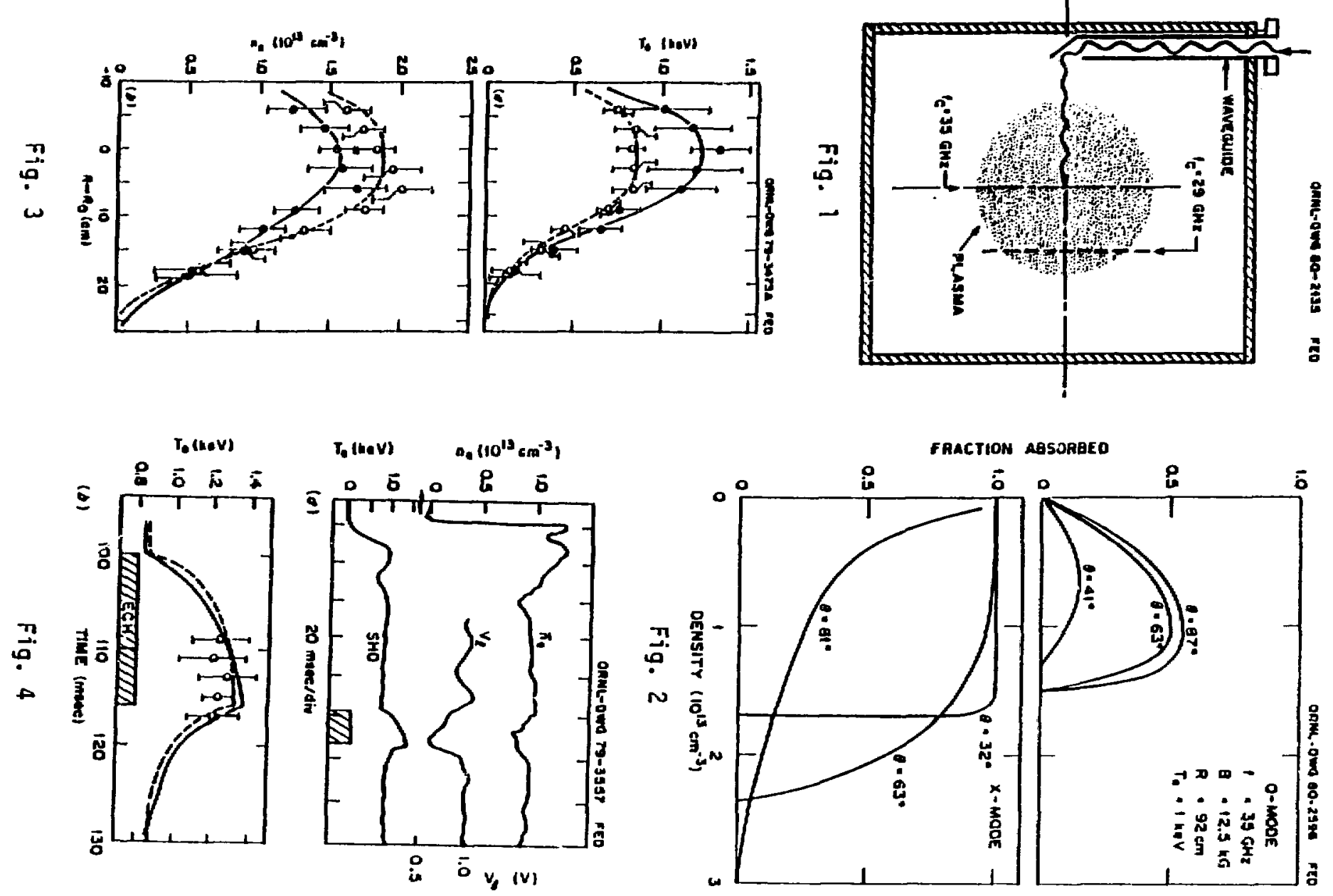

$\infty$ 


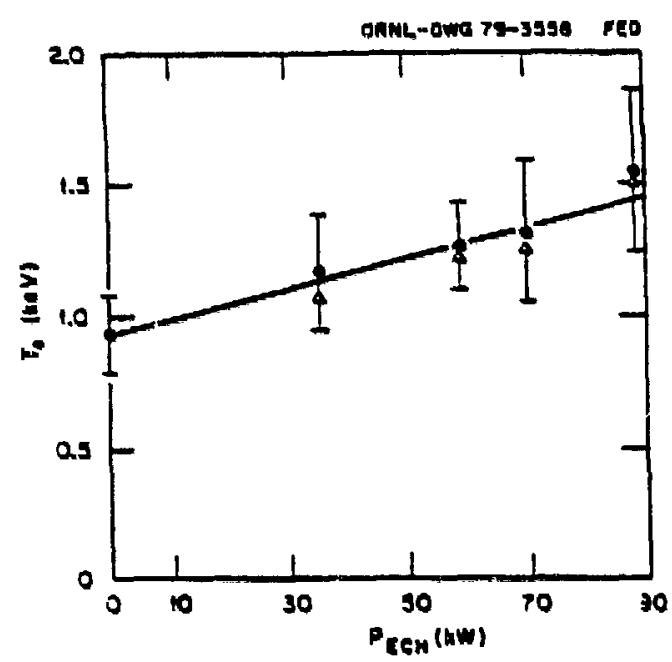

Fig. 5

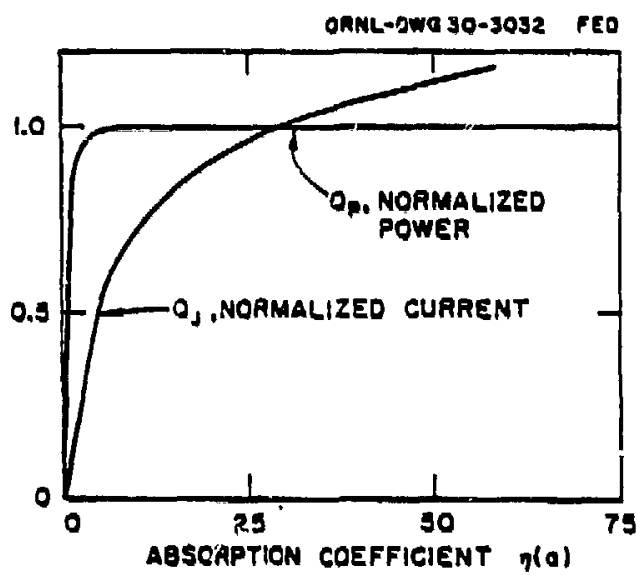

Fig. 6

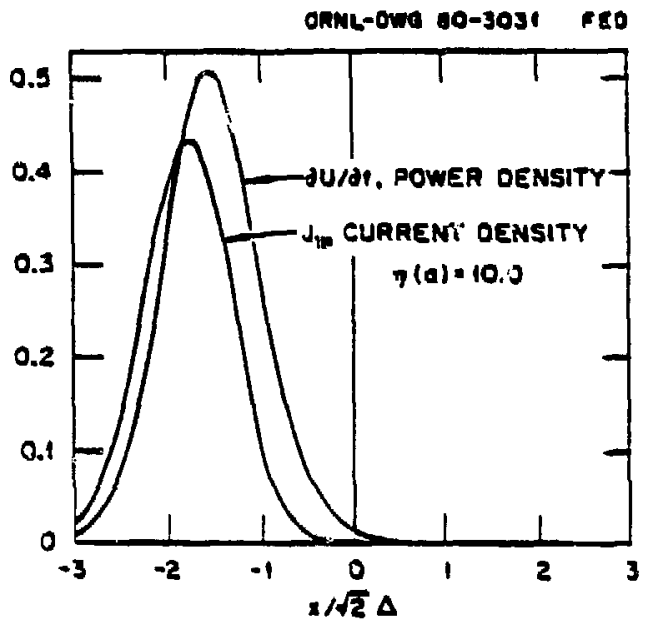

Fig. 7

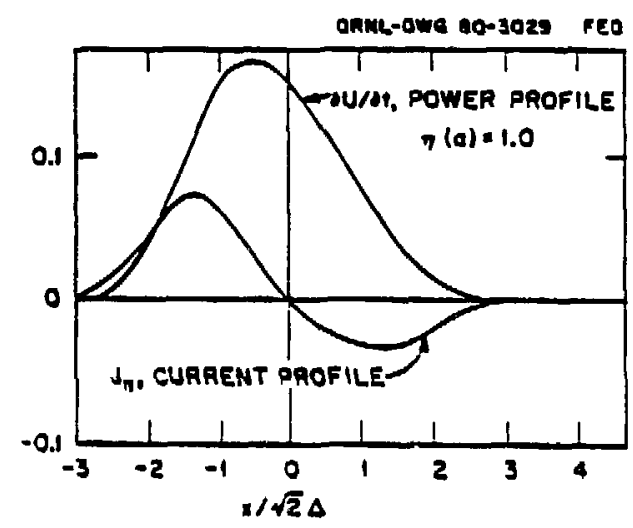

Fig. 8 